\title{
Die Königskrönung Wratislavs von Böhmen und die angebliche Mainzer Synode des Jahres 1086. Von
}

\section{H. Spangenberg.}

Von Mähren, Polen und Böhnen aus war seit dem Niedergange der karolingischeu Herrschaft die Begründung eines selbständigen Slavenreiches an der Ostgrenze Deutschlands versucht worden mit steigendem Misserfolge, je mehr das abendländische Kaiserreich au Macht und äusserer Ausdehnung gewann. Die umfassenden Pläne, welche Suatopluk von Mähren der Verwirklichung nahegefülırt, der Pole Boleslav Chabri mit minderem Glücke aufgenommen, scheiterten in Bretislars Hand an dem Widerstand Kaiser Heinrichs III. Der panslavistische Gedanke hatte sich überlebt, seit die Staatenbildungen im Osten des Reichs zu fester Gestalt gelangt und durch die beherrschenden Mächte des Abendlandes dem Organismus des Reichs und der katholischen Kirche eingefügt waren.

In richtiger Erkenntnis der politischen Lage und der Schranken seiner eigenen Macht gab der Premyslide Bretislar nach dem missglückten Feldzuge des Jahres 1041 den Widerstand gegen die Reichsgewalt auf und bewahrte seitdem Heinrich III. die Lehenstreue. Der Sohn und Nachfolger des mächtigen Kaisers fand in der Zeit des tiefen Verfalles des fränkischen Königthums in Herzog Wratislav von Böhmen die zuverlässigste Stütze seiner Herrschaft. Bei dem schroffen Gegensatz zu Polen, der feindlichen Gesinnung seiner Brüder, der Widersetzlichkeit des böhmischen Adels und Clerus hatte Wratislav eiu wohlbegründetes Interesse, sich Heiurich IV. zu verbinden, um so mehr als dieser ihm eine Vergrösserung seines Herrschaftsgebietes 
durch Erwerb der Mark Meissen in Aussicht stellte 1). Nach der Sachsenschlacht bei Homburg (9. Juni 1075) erfüllte der König sein Versprechen; aber kurz darauf mussten die Böhmen dem jugendlichen Ekbert von Meissen und seinen sächsischen Bundesgenossen die östlichen Marken wiederum räumen ${ }^{2}$ ).

Herzog Wratislav blieb nur der Anspruch auf die sächsischen Grenzmarken. Die Ungunst der Zeiten rersagte es ihm, sich mit Waffengewalt in den Besitz der verlorenen Gebiete zu setzen; denn in den traurigen Zeiten, da die Bande des Reichs sich allerorten lösten, der polnische Herzog sich die Königskrone aufs Haupt setzte, Ungarn die Fesseln deutscher Herrschaft abschüttelte, stand der Böhme unter den Freunden des Königs fast vereinsamt. Von Heinrich IV. durfte er keine Hülfe erwarten, seit das schuldbeladene deutsche Fürstenthum dem Papste die Hand reichte, um das Königthum der tiefen Erniedrigung von Canossa zu unterwerfen.

Als Heinrich IV. vom Banne gelöst wach Deutschland zurückkehrte, gesellte sich Wratislav zu den wenigen Getreuen, die sich Anfang 1077 am Regensburger Hof um ihren schwer gedemüthigten Herrscher scharten ${ }^{3}$ ). Seitdem stand er ihm zur Seite ausdauernd und opferfreadig, wie kein anderer Reichsfürst. Böhmische Truppen kämpften 1077 in Schwaben ${ }^{t}$ ) und Baiern 5); bei Melrichstadt am 7. August 1078 deckten sie den Rückzug der geschlagenen kaiserlichen Truppen ${ }^{6}$ ); in der Schlacht bei Dorla (am 27. Jan. 1080) sollen 3255 Böhmen in heldenmüthigem Kampfe gefallen sein, unter.ihnen der

1) Bruno De bello saxonico M. G. V 341, 342. Mit Giesebrecht Geschichte der deutschen Kaiserzeit III 2 S. 1137 an der Thatsache jenes Versprechens zu zweifeln, scheint mir kein Grund vorzuliegen. Ekbert war zwar 1074 im Besitz Meissens und stand, wie es scheint, auf Heinrichs [V. Seite. Das hinderte aber den König nicht, 1075 in Ekberts Besitzungen einzufallen und einen Theil derselben seinem Günstling Udalrich von Godesheim zu schenken cf. Bruno cap. 56 M. G. V. 349 ; und 1076 hat der König dem böhmischen Herzog das Versprechen thateächlich erfüllt, das er ihm nach Bruno's Bericht zwei Jahre zuvor gegeben haben soll cf. Lamberti ann. M. G. V 233.

2) Lamberti ann. M. G. V 249, 250.

3) Bertholdi ann. M. G. V 294.

4) Bertholdi ann. M. G. V 295; Bernoldi chron. M. G. V 434; Bruno De bello saxon. M. G. $\nabla$ 367. Ueber Wratislavs Theilnahme am Hoftag von Nürnberg vgl. K. F. Stumpf Die Reichskanzler Nr. 2802 (Urk. v. 11. Juni 1077).

5) Bertholdi ann. M. G. V 302.

6) Chron. petershusanum II 34 bei F. J. Mone Quellensammlung der badischen Landesgeschichte Karlsruhe 1848 Bd. I 137. 
Burggraf von Prag ${ }^{1}$ ); auch in der Elsterschlacht bei Hohen-Mölsec hatte Heinrich IV. auf Wratislavs Hülfe gerechnet ${ }^{2}$ ).

Der Versuch der Böhmen, während des deutschen Bürgerkrieges: die Meissner Mark in ihren Besitz zu bringen (1079), missglückte ${ }^{3}$. Ein Ersat\% schien sich zu bieten, als Heinrich IV. den Babenberger Liutpold, welcher 7.u Tulu dem hönige die Treue abgeschworen und dessen Anhänger aus deru Lande vertrieben. die österreichische Mark aberkannte. Sie wurde - vermuthlich während des Regensburger Hoftages im März 10;1 - dem Yřemysliden übertragen ${ }^{4}$ ).

Indesseu Bořivoy. Wratislavs jugendlicher Sohu, unter der Ubhut Wiprechts von Groitsch. eines waffengeübten und den Böhmen seit längerer Zeit dienstbaren Rittersmannes den deutschen Kaiser nach Rom geleitete 5), versuchte der Herzog sich in den Besitz der österreichischen Mark zu setzen. Am 12. Mai 1082 erfocht er bei Mailberg nahe der mährischen Grenze, wo Böhmen und Oesterreicher sich damals zum ersten Male in blutigem Kampfe massen. einen entschiedenen Sieg $\left.{ }^{6}\right)$. A ber obwohl Heiurich IV. selbst 1084 nach der Rückkehr aus Italien den Kampf gegen den Abtrünnigeu erneuerte '), blieb der Babenberger in Besitz der österreichischen Mark.

Erst spät erhielt der Herzog den längst verheissenen Lohn ausdaueruder Treue. Auf der Synode zu Mainz (nach Cosmas 1086)

1) Bertholdi ann. M. (i. V 324, 325: Bruno De bello saxon. M. (i. Y 378.

2) Nach Bruno M. (i. I' 3\$0, 381, der über die Ereignisse in deutschen Norden gut orientirt ist und. wie es scheint, persönlirh an der Schlacht bei Mölsen theilnahm (vgl. hap. 13), wartete der König rergeblich auf die Ankunft der Böhmen. Die Pegauer Annalen M. G. XVI 241, 24\%. eiue wenig zuverlässige Quelle, berichten, dass Wratislav und Wiprecht von Groitsch am Kampfe theilnahmen und dem geschiagenen kaiserlichen Heer das Geleit durch Böhmen gaben : und nach der Petershauser Chronik Il 38 bei Mone a. a. O. I 137 waren die Operationen des Gegenköniqss Rudolf ausschliesslich gegen Wratıslav und sein slavisches Kriegsheer gerichtet.

s) Bertholdi ann. M. (x. Y 320 ; ann. pegav. M. G. XVI 241.

4) Vita Altmanni cap. 25 M. G. XII 236. Dass Wratisiav im März 1081 sich in des Königs Gefolge befand, bezengt Stumpt Acta imperii S. 77, 78 Ni. 74 (Urk. v. 18. März 1081). Vgl. U. Posse Die Markgrafen von Meissen und das Haus Wettin. Leipzig 1881 今. 188 Ann. 102.

5) Ann. peg. M. G. XVI $238-240$.

ค) Vita Altmanni M. G. XII 236; ann. mellic. M. G. IX 500; contin. claustroneob. M. G. IX 608. Dass die Darstellung des Cosmas II 35 M. G. IX 90 grösstentheils einem Schlachtbericht Regino's von Prümm (zum Jahre 891) entlebnt und daher unbrauchbar ist, bemerkt Bretholz Geschichte Mährens I 2,210 Anm. 1.

7) Ann. iburg. M. G. XVI 438. 
wurde er von Heinrich IV. mit Zustimmung der anwesenden weltlichen und geistlichen Fürsten zun Könige, von Böhmen und Polen" emanut, und in des Kaisers Auftrage rom Erzbischof Egilbert von Trier zu Prag am 15. Juni mit seiner Gattin Suatava feierlichst gekrönt 1 ).

So erzählt Cosmas von Prag, der uns von der Krönung des Böhmen ausführlicher Nachricht gegeben hat. Auch er ist oberflächlich genug unterrichtet; nichts desto weniger hat er allgemein Glauben gefunden. Nach übereinstimmender Annahme Palacky's, Dudiks, Giesebrechts u. a. *) sind nach Heinrichs IV. Rückkehr vom Romzuge (1084) zwei Synoden zu Maiuz in den Jahren 1085, 1086 abgehalten worden. Ueber den Verlauf der ersteren sind wir durch eine grössere Anzahl zuverlässiger

1) Es wäre möglich, dass der Rechtstitel eines Königs von Polen aus der Trihutzablung hergeleitet wurde, zu welcher die Polen Böhmen gegenüber tür die ihnen 10jt von Bretislar abgetretenen Länder verpflichtet waren (cf. ann. alt. M. G. XX 807; Cosmas II 13 M. G. IX 75, III 1 M. G. IX 102). Die Anschauung, dass diese Tributzahlung eine Art Abhängigkeit Polens begründe, spricht sich z. B. auch in den Worten aus. welche Cosmas ILI 3ti M. G. IX 120 Wladislar in den Mund legt, um die Brhmen (1110) zum Kampf gegen Polen aufzumuntern: , O Boemi ..... Nunc vestri tributarii, quibus semper fuistis timori. vobis adhuc spirantibus insultant et terram vestram devastantc. Indessen erscheint es mir zweifelhaft, ob dem Přemysliden thatä̈chlich, wie Cosmas allein behauptet, der Titel eines Krnigs von Polen beigelegt wurde: Die rom Cbronisten mitgetheilten Worte der Acclamation der Böhmen nach Wratislavs Krönung stimmen fast wörtlich (vergl. Manitius in den Mittheil. d. Inst. f. österr. Gesch. 1887 VIII 482) mit dem Zuruf der Römer bei Karls des Grossen Kaiserkrönung überein, sind also vermuthlich einer fränkischen Quelle entlehnt. Und ferner ist. es bemerkenswert. dass sich der Titel, rex Polonorum “ für Wratislav uxkundlich nirgends nachweisen lässt. Vgl. auch Röpell Geschichte Polens I 208. Hiernach ist es nicht unwahrscheinlich, dass der Anspruch Böhmens auf einen Vorrang dem feindlichen polnischen Schwesterstamme gegenüber Veranlassung war zur Entstehung der ron Cosmas übermittelten Tradition, dass Wratislav $108 j$ auch zum König von Polen ernannt sei.

2) Palacky Geschichte Böhmens I 318 ff.; Dudik Geschichte Mäbrens II 420 ff. ; Giesebrecht Geschichte der deutschen Kaiserzeit III 606 ff., 616 ff.; H. Floto Kaiser Heinrich IV. und sein Zeitalter 1856 Bd. II 318: G. A. Stenzel Geschichte Deutschlands unter den fränkischen Kaisern. Leipzig 1827 S. 527 etc. Wenn F. Schannat und J. Hartzheim Concilia Germaniae Tom. III 1760 S. 201-203 die Vereinigung der Bisthümer Prag und Olmütz zum Jahre 1085 berichten, so ist diese Angabe wohl aus einem Versehen der Herausgeber zu erklären. Alle späteren Darstellungen der Conciliengeschichte führen zwei Mainzer Synoden (1085 und 1086) an, so Mansi Sacrorum conciliorum collectio 1775 Tom. XX S. 603, 613 ff., $635 \mathrm{ff}$; C. J. von Hefele Conciliengeschichte Freiburg $1886 \mathrm{Bd}$. V $182-183,183,186-187$ น. a. 
Quellen unterrichtet: von der zweiten dagegen, währeud deren Wratislav's Krönung stattgefundeu haben soll, berichtet Cosmas allein. Um die Richtigkeit seiuer Angabe prüfen zu können, stellt sich zunächst die Frage, ob die Darstellung des Chronisten im allgemeinen Glaubwürdigkeit beanspruchen darf.

Im zweiten Buch seiner Chronik Cap. 37 erzählt Cosmas, auf der Mainzer Synode des Jahres 1080 sei Wratislav von Heinrich IV. zum Könige Böhmens gekrönt worden. Während derselben Synode habe Gebhard, der Bischof von Prag, seine Klageschriften gegen Johanu, den Bischof vou Mähren, vorgelegt. Dieser sei im Laufe des Jahres gestorben, doch habe Gebhard in kluger Sorge für die Zukunft das Ohr des Kaisers durch seine Freunde zu gewinneu versucht, damit nicht ein neuer Bischof von Olmütz ernannt würde. Um seinen Anspruch auf Vereinigung der Diözesen PragOlmütz zu begründen, habe er Heinrich IV. ein Privileg vorgelegt -privilegium olim a sancto Adalberto episcopo, suo antecessore, confirmatum tam a papa Benedicto quam a primo Ottone imperatore. Ad cuius iustam querimoniam imperator notus precibus ducis Wratizlai fratris eiusdem episcopi Gebeardi, et consilio archiepiscopi Maguntini Wezelonis et aliorum bonorum, qui iusticiae favehant, novum antiquo fere eiusdem tenoris addit privilegium et signo imperiali confirmat, ut in sequentibus patebit". Es folgt der Wortlaut des Diploms Heinrichs IV. vom 29. April 1086.

Mit dem erwähnten „Privileg“ Otto's I. hat Cosmas 2 weifellos die Stiftungsurkunde des einst von Kaiser Otto I. und Papst Benedict VI. begründeten Bisthums Prag gemeint. Om die Echtheit dieses Privilegs ist ein heisser Kampf gelührt worden. Nachdem Loserth 1) in Uebereinstimmung mit Huber ${ }^{2}$ ) versucht, die angebliche Vorlage des kaiserlichen Diploms als Fälschung Bischof Gebhards zu erweisen, Kalousek ${ }^{3}$ ) Losertbs Einwände gegen die Echtheit desselben bekämpft hatte, ist von $B$. Bretholz ${ }^{4}$ ) überzeugend nachgewiesen worden, dass in dem neuen von Cosmas mitgetheilten Diplom Heinrichs IV. vom 29. April 1086 weder eine echte noch eine gefälschte Crkunde aufgenommen ist und die Benutzung einer Vorlage sich höchstens auf die Beschreibung der Prager Bisthumsgreuzen beziehen kann. Diese

1) Loserth, Der Umfang des böhmischen Keiches unter Boleslavs in den Mittheil. d. Inst. f. österr. Gesch. II 17-28.

2) Mittheil. d. Inst. f. österr. Gesch. II 385.

s) Sitzungsberichte d. königl. Ges. der Wissenschaften zu Prag 1883 S. 26 ff.

4) Bretholz , Mähren und das Reich Herzog Boleslavs II. von Böhmen " im Archip f. österr. Gesch. 1895 Bd. 82 S. 139 ff. 
aber nimmt in dem kaiserlichen Diplom nur etwa deu vierten Theil des Textes ein. Alles übrige in demselben ist „durchans neue, den Zeitumständen und momentanen Verhältnissen angepasste Fassuug: 1). Die Behauptung des Cosmas ist daher sicherlich falsch, das neue Diplom babe mit dem alten übereinstimmend ("fere eiusdem tenoris") gelautet. Auch ist es mindestens unwahrscheinlich, aass Bischof Gebhard der Synode zur Begründung seiner Ansprüche überhaupt ein Privileg Otto's I. vorgelegt hat. Die echte Stiftungsurkunde konnte er keinesfalls für seinen Zweck verwerten. Denn da zur Zeit der Prager Bisthumsgründung Mähren kirchlich selbständig ${ }^{2}$ ) und politisch von Böhmen getrenut war ${ }^{3}$ ), wäre sie eher geeignet gewesen, die

1) Vgl. Bretholz a. a. O. S. 158.

2) In einer Urkunde des Erzbischof's Willigis ron Mainz vom 28. April 976 (Boczek Codex Moraviae dipl. I 97) werden ausser anderen Suftraganen des Mainzer Stuhles die Bischöfe von Böhmen und Mähren (sepiscopus Moraviensise) als Zeugen einer Gerichtshandlung aufgetührt. Die Existenz eines mährischen Bischofs in der Zeit vor Gründung des Olmützer Episkopates wird durch die Nachricht des Cosmas II 21 M. G. IX 80 bestätigt, dass Măhren schon vor der Zeit Severs von Prag (1031-1067) einen Bischof gehabt habe, quidam episcopus, ut reor nomine Wracen". Ausserden wird im Granum catalogi pracsulum Moraviae ein Bischot Silvester von Mähren genant. Mehr ist freilich inber das Bestehen eines mährischen Bisthums im zehnten Jahrhundert nicht bekannt. Die Annahme Dudiks II 46 ff., dass del bei Boczek I 97 erwăhnte episcopus Moraviensis eir dem Prager Bischof zur Aushülfe beigeordneter Weihbischof gewesen sei, weist Bretholz (Archiv f. österr. Gesch. Bd. 82, 155) mit gutem Grunde zurück. Vgl. A. Hauck Die Kirche Deutscblands. Leipzig 1896 Bd. III $199 \mathrm{ff}$. Anm. 4.

ง) Die ron Palacky I 221, Dudik II 13 u. a. vertretene Ansicht, dass Mähren schon vor der Prager Bisthumsgründung von den Přemysliden zurück. erobert worden sei, hat Bretholz a. a. O. S. $139 \mathrm{ff}$. widerlegt. Sie steht unter anderem im Widerspruch zu der ausdrücklichen Angabe des Cosmas I 40 M. G. IX 63, dass Herzog Bretislar (1034-1055) zuerst, primus Mähren mit dem böhmischen Reiche wieder verbunden habe: ,posteri sui discant, quod terra Moravia et eius dominatores semper Boemorum principis sint sub potestate, sicut avus noster piae memoriae Bracizlaus ordinavit, qui eam primus dominio suo subiugavit.

Mähren blieb im zehnten Jahrhundert unter ungarischer Herrachaft, bis es nach Boleslavs II. Tode (999) von den Polen erobert wurde. Den Zeitpunkt dieses Ereignisses deutet Cosmas I 40 M. U. IX 63 an: ,post obitum secundi Bolezlai sicut urbem Pragam, ita totam Moraviam vi obtinuerant Poloniic. Ein sicherer Terminus ante quem für Mährens Unterjochung durch Boleslav Chabri wird durch Thietmars Mittheilung VII 42, 44 M. G. III 854, 856 gegeben, dass Mähren im Jahre 1017 auf Polens Seite gegen Böhmen kämptte.

Nach der Zeit ungarischer und polnischer Herrschaft wurde Mäbren im Jahre 1029 durch Bretislav, den Sohn Herzog Udalrichs, dem böhmischen Reiche 
Ansprüche Gebhards auf Mähren zu widerlegen, als sie zu begründen. Hätte Gebhard thatsächlich den Synodalen ein Privileg Otto's I. vorgelegt und aus diesem seine Rechtsforderungen abgeleitet, so würde in dem Diplom Heinrichs IV. hierauf wohl Bezug genommen sein. Dies geschieht aber keineswegs. Es hat rielmehr den Anschein. dass Cosmas der Berufung Gebhards auf die ottonische Gründungsurkunde eine Bedeutung beigemessen, die sie in Wirklichkeit in den Mainzer Verhandlungen nicht gehabt hat. Den Nittelpunkt derselben bildete Gebhards Beschwerde über Gründung des Olmützer Bisthums (1063) 1) nnd die hiermit verbundene Trennung Yährens rom böhmischen Episcopate. Um diese als unrechtmässig zu erweisen, scheint Gebhard freilich unter anderen drgumenten auch geltend gemacht zu haben, dass Böhmen und Mähren von Anfang an kirchlich zu einander gehörten; und dies war richtig, wenn man es auf die Zeit seit der politischen Vereinigung beider Länder durch Bretislavs Eroherung (10:(1) bezieht.

Es ist sehr wohl müglich. dass die Darstellung des Cosmas .replicat coram omnibus privilegium olim a sancto Adalherto episcopo, suo antecessore, confirmatum tam a papa Benedicto quam a primo Ottone imperatore“ im Grunde nur auf den folgenden Worten des kaiserlichen Diploms beruht: "conquestus est (scl. Gebeardus), quod Pragensis episcopatus, qui ab initio per totum Boemiae ac Moraviae ducatum unus et integer constitutus, et tam a papa Benedicto quam a primo Ottone imperatore sic confirmatus est. . . . . . divisus esset et imminutus". Dies ergibt sich. wie mir scheint, auch aus dem auffallenden Widerspruch der Angaben des Chrouisten an obiger Stelle (II 37) mit denen des ersten Buchs "), in welchem er die Griindung des Prager Bisthums erzählt. Hier erwähnt er weder Kaiser Otto noch Papst Benedict, sondern führt die Stiftung des Episcopats lediglich auf Papst Johann XIII. und die Initiative Herzog Boleslavs II. zurück. Es liegt die Vermuthung nahe, dass Cosmas, der Prager Domdekan, sich durch Eifer für das Wohl seiner Kirche zu falschen Angaben oder mindestens tendeuziöser Entstellung seiner Quelle hat verleiten lassen.

unterworfen. Vgl. Dudik II $164 \mathrm{ff}$; Bresslau Jahrbb. des deutschen Reichs unter Konrad II. Bd. I 267 Anm. :2.

1) Das Gründungsjabr des Olmützer Bisthums ist genannt im Granum catalogi praesulum Moraviae, herausgegeben von J. Loserth im Archiv f. österr. Gesch. Bd. 78; vgl. daselbst S. 67.

2) I 22 M. G. IX 49. 
Unhistorisch ist es, wenn Cosmas in den citirten Worten „privilegium olim a sancto Adalberto episcopo, suo antecessore, confirmatum tam a papa Benedicto quam a primo Ottone imperatore", die in der vorliegenden Fassung nicht gan $\angle$ verständlich sind, dem beiligen Adalbert Betheiligung an der Prager Bisthunsgründung zuschreibt. Es stimmt dies freilich mit seinem chronologischen System überein, nach welchem der Tod Thietmars, des ersten Prager Bischofs 1), und auch die Wahl Adalberts ${ }^{2}$ ) im Jahre 969 d. i, noch zu Lebzeiten Otto's des Grossen erfolgt ist. Thatsächlich wurde Adalbert 982, fast zehn Jahre nach Otto's I. Tod, Bischof. Er kaun daher als solcher an ler Stiftung des Bisthums Prag: die spätestens 976 anzusetzen ist ${ }^{3}$ ). nicht betheiligt gewesen sein.

Wenn Cosmas ferner berichtet, Wratislav selbst habe den Kaiser gebeten, seinem Bruder Gebhard das neue Privileg auszustellen, so kann auch dies nicht dem wirklichən Verlauf entsprechen. Wratislav hatte einst seine ganze Kraft autgeboten, um das Olmützer Bisthum ins Leben zu rufen. Nur durch sein dringendes Verlangen („nimia devictus efflagitatione Wratizlai ducis $\left.{ }^{4}\right)^{4}$ ) war Bischof Sever von Prag beredet worden, in die Ernennung Johanns zum mährischen Bischof zu willigen. Wratislav's ganzes Herz hing an der neuen Schöpfung; in hartem Kampf mit seinem Bruder Jaromir (als Bischof ,Gebhard" genannt) schützte und vertheidigte er sie. Und als sie durch Heinrichs IV. Diplom rom 29. April 1086 aufgehoben wurde, stellte er Gebhard so lange nach, bis dieser aus Böhmen floh und die Selb-

1) Thietmar wurde zwischen dem 25. Jan. 975 und dem 28. April 976 zum Bischof geweiht rgl. Köpke-Dümmler Otto der Grosse S. 503 Anm. 2.

2) Nach der Adalbertsbiographie des Canaparius M. G. IV 584 Cap. 8, den sich Cosmas I 26 M. G. IX 50, 51 fast wörtlich anschliesst, ist Adalbert während Otto's II. Aufenthalt in Verona (Mai, Juni 983) investirt worden. Cosmas setzt das genauere Datum, den 3. Juni, hinzu. Damit ist für die Wahl ein terminus ante quem gewonnen. Cosmas I 25 M. IX G. 50 hält es für nöthig, ausdrücklich zu betonen, dass die Wahl Adalberts am 19. Februar desselben Jahres stattfand, in welchem 'Thietmar, sein Vorgänger, starb. Thietmar starb im Jahre 982. Im übrigen vgl. H. G. Voigt Adalbert von Prag. 1898 Anm. 177. Die böhmischeu Annalen (b. Miklosich Slavische Bibliothek II 302) berichten die Consecration, welche thatsäcblich am 29. Juni 983 stattfand, zum Jabre 982 (972 ist aus 982 verschrieben, wie aus den vorangehenden und folgenden Zahlen leicht ersichtlich). Vermuthlich haben sie das Jahr der Wahl mit dem der Consecration verwechselt.

s) Nach Körpke-Dümmler Otto der Grosse. Leipzig 1876 S. 503 fand die Gründung des Bisthums Prag 975 oder 976, nach A. Hauck Die Kirche Deutschlands. Leipzig 1896 Bd. III 199 im Jahre 975 statt. Vgl. Giesebrecht Geschichte der deutschen Kaiserzeit $1881 \mathrm{Bd}$. I 847 u. a.

4) Cosmas II 21 M. (i. IX 80 . 
ständigkeit der mährischen Kirche durch Erneunung eines Oluützer Bischofs erneuert werden konute 1). Und doch soll der Kaiser, wie Cosmas behauptet, das erwähnte Diplom ,motus precibus Wratialai* ausgestellt baben! Nach den Worten des kaiserlichen Diplom's geschah es vielmehr ,assensu ... ducis Boemorum Wratizlai* und nach Vermittlung der Fürsten: . Vediantibus itaque nobis et communi principum aspirante suffragio factum est, ut dux Boemiae Wratizlaus et frater eius Chounradus supradicto Pragensi episcopo, fratri suo, parrochian iudiciario ordine requisitam ex integro et reprofiterentur et redderent". Auch hier ist aus der Art, in welcher Cosmas deu Text seiner Chronik gestaltet, eine bestimmte Tendeuz deutlich zu erkennen. Wie er an der Berechtigung der Klage Gebhards keinen Zweifel aufkommen lassen wollte und sie auf cin Privileg Kaiser Otto's I. und Papst Benedicts begründete, so geuügte ihm nicht, dass Wratislav nothgedrungen seine Zustimmung zur Vereinigung der Diöcesen Prag und Olmütz gab, sie sollte vielmehr auf Antrag des Herzogs selbst geschehen sein.

Ein weiteres Moment kommt hinzu, die Glaubwürdigkeit des Cosmaz zu erschüttern. Wer die wenigen eben besprochenen Sätze des Capitels $37 \mathrm{im}$ Zusammenhange liest, gewinnt nach ihnen den bestimmten Eindruck, als sei das Privileg vom \&9. April 1086 während der Mainzer Synode vom Kaiser unterzeichnet worden. Sie beginnen: "In eodem concilio Pragensis praesul Gebeardus scripta suae antiquae querimoniae representat" etc. Die alte Stiftungsurkunde soll vorgelegt. seiu "coram omnibus" d. i. in Gegenwart der zu Mainz Versammelten; Gebhard, Wratislav, Erzbischof Wezelo, durch deren Verwendung sich Heinrich IV. nach Cosmas bestimmen liess, das Privileg vom 29. April zu unterzeichnen, werden vom Chronisten ansdrücklich als Theilnehmer an den Synodalverhandlungen aufgeführt. Wenn Cosmas daher hinter dem Text des Privilegs bemerkt: „quod (scl. signum) ego vidi ipsum caesarem suis manibus annotantem in privilegio Pragensis episcopatus $\left.{ }^{4}\right)^{2}$ ), so kann man nach der gesammten Darstellung nicht anderer Meinung sein, als dass er selbst den Kaiser in Mainz das Signum habe zeichnen sehen. In dem Text der Urkunde, welche Cosmas bis zur Signumzeile einschliesslich in die Chronik aufgenommen, ist der Ausstellungsort nicht mehr genannt. Eine im Münchener Reichsarchiv befindliche

1) Cosmas II 41 M. G. IX 95. Der böhmisch-mährische Kirchenstreit ist von Palacky, Dudik, Giesebrecht u. a. ausführlich dargestellt worden. Einen wichtigen Beitrag lieferte neuerdings J. Lippert, Die Wyschebradfrages in den Mittheilungen des Vereins f. Gesch. d Deutscben in Böhmen 1894 Bk. 3\%, $213 \mathrm{ft}$.

2) M. G. IX 93. 
Copie der Urkunde, welche vermathlich aus dem zwölften Jahrbundert stammt, schliesst nun mit den Worten ,Actum Ratispone in Christi nomine feliciter amen " 1 ). Demnach ist das Diplom in Regensburg ausgestellt, nicht in Mainz. Durch diese Thatsache erscheint des Cosmas Angabe, er habe den Kaiser das Signum zeichnen sehen nämlich zu Mainz, wie man nach seiner Darstellung annehmen muss - in höchst eigenthümlichem Licht. Warum liess er in seiner Abschrift allein die Actumzeile aus? Hatte er etwa ein Interesse daran, die Verhandlungen über Aufhebung der mährischen Kirche vom Anfang bis zuin Ende unter die Sanction der Mainzer Synode zu stellen? Oder hat er sich nur geirrt? Hatten die Ereignisse sich in seinem Gedächtnis so sehr verwischt, dass er nur erinnerte, bei der Unterzeichnung des Kaisers zugegen gewesen zu sein, dagegen vergass, ob dies in Mainz oder Regensburg geschehen?

Wie dem auch sei, es ist deutlich, dass die kurze Darstelling des Capitels 37 eine verhältnismässig grosse Anzahl falscher oder entstellter Angaben aufweist und der Autorität, die der Chronist als angeblicher Augenzeuge beansprucht, kein Wert beizumessen ist. Man wird hiernach geneigt sein, auch in die Existenz der Mainzer Synode vom Jahre 1086, die Cosmas allein erwähnt, Misstrauen zu setzen. Zwei wesentliche Argumente ergeben sich hierfür: 1. aus dem Inhalt des Diploms rom 29. April 1086 und 2. aus dem Itinerar Kaiser Heinrich's IV.

1. Dass das kaiserliche Diplom nicht in Mainz vollzogen worden, wird durch den Inhalt desselben bestätigt; in ihm wird von der Mainzer Synode als von einem vergangenen, geschichtlichen Ereignis gesprochen: "Qui (scl. Gebeardus) cun Magunciae coram legatis apostolicae sedis . . . querimoniam intulisset, . . . . primitiva illa parrochia cum omni terminorum suorum ambitu Pragensi sedi est adiudicata". Und nach den Worten "Mediantibus itaque nobis et communi principum aspirante suffragio factum est, ut dux Boemie Wratizlaus et frater eius Chounradus supradicto Pragensi episcopo, fratri

1) Stumpf Acta imperii Nr. 76 (Die Reichskanzler Bd. III 79--81). Das vollständige Schlussprotokoll der Urkunde lautet: , Signum domini Henrici tertii imperatoris augusti. Hermannis cancellarius vice Wezelonis archicancellarii recognovit. Datum III kalendas Maii anno ab incarnatione domini MLXXXVI, indictione VIII, anno autem domini Henrici regni quidem XXXII, imperii vero III. Actum Ratispone, in Christi nomine feliciter amen «. Da im 'Text des Diploms ausdrücklich vermerkt wird, dass die, Handlung in Mainz stattfand, sind, actum" und, datum" auf die Beurkundung zu beziehen; vgl. Ficker Beiträge zur Urkundenlehre Bd. I 131, 132 Nr. 87 ; Bresslau Handbuch der Urkundenlehre 1889 Bd. I $856 \mathrm{ff}$. 
suo, parrochiam iudiciario ordine requisitam ex integro et reprofiterentur et redderent" hat es den Anschein, als sei nicht nur der Synodalbeschluss, auch die thatsächliche Vereinigung des böhnischen und mährischen Bisthums zur Zeit, da die Crkunde vollzogen wurde, bereits erfulgt. Die Mainzer Synode müsste also geraume Zeit vor dem 29. April 1086 stattgefunden baben.

2. Diese Erwägung wird dadurch bestätigt, dass bei der Bestimmung des Itinerars Heinrichs IV. im Frühjahr 1086 kein Platz für eine Mainzer Synode bleibt: Am 27. Januar ${ }^{1)}$ zog (ler Kaiser gegen die Sachsen zu Felde; am 7. Februar hielt er zu Wechmar über Ekbert von Meissen ein Fürstengericht ${ }^{2}$ ), um kurz darauf nuch vor dem Aschermittwoch (18. Februar) ${ }^{3}$ ) das Herzogthum zu verlassen. Da er am 3. 9., 29. April in Regenshurg nachweisbar ist ), haben Giesebrecht 5), Huber ${ }^{6}$ ) u. a. die Synode in den März 1086 verlegt; doch widerspricht ihre Annahme dem ausdrücklichen Zeugnis der wohlunterrichteten, noch zu Heinrichs IV. Zeit entstandeneu Augsburger Annalen: „Imperator .... exercitus multitudine collecta iterum Saxoniam invadit; sed adversariis in diversa cedentibus, ipse partem provintiae devastat, incendit .....; qui etiam statim in Pauwariam eo reverso, coniurationis suae assumptis fautoribus, Frisingam, seducto cum dolis episcopo, in paschali sollemnitate (5. April) capiunt" 7). Da der Kaiser "sogleich" nach Verwüstung Sachsens in das bairische Herzogthum zurückkehrte, so bleibt nur übrig. die angebliche Synode von 1086 in ein früberes Jahr zu rücken.

Erweist sich die Chronologie des böhmischen Chronisten aus den angeführten Gründen als unrichtig, so liegt die Vermuthung nahe, dass die Schilderung des Cosmas zu 1086 sich thatsächlich auf die Mainzer Syuode des Jahres 1085 bezieht, von der auch die deutschen Annalisten berichten. Und mit dem Verlauf der Letzteren stimmt die Darstellung des Cosmas bis auf geringe Differenzen überein!

1) Bernoldi chron. M. G. V 444.

2) Stumpf Die Reichskanzler Nr. 2879.

s) Liber de unitate ecclesiae conservanda ell. W. Sihwenkenbecher II 29 S. 98. (Schulausgabe der M. G. Hannover 188:3).

4) Stumpf Die Reichskanzler Nr. 2880-288\%. Nach Bernolds Chronit M. G. V $44 \pm$ war Heinrich am 5. April in Regensburg eingeschlossen.

5) Geschichte der deutschen Kaiserzeit III 616 .

$\left.{ }^{B}\right)$ Geschichte Oesterreichs I 232. J. Kröger Geschichte Böhmens rom Friedensschluss Bretislavs mit Heinrich III. (1041) bis Wratislavs Königskrönung Diss. Leipzig $1880 \mathrm{~S} .65$ verlegt die Krönung in den, Winter ${ }^{\star} 1086$.

7) M. G. III $131,132$. 
Cosmas spricht von einer ,sinodus magna in urbe Maguntia, ubi 4 archiepiscopi et 12 praesules . . . plurima decreta super statu sauctae ecclesiae scriptis roboraverunt" ${ }^{1}$ ). Es wäre höchst seltsan, wenn 1086 eine so bedeutende, zahlreich besuchte und durch umfangreiche gesetzgeberische Thätigkeit ansgezeichnete Synode stattgefunden bütte, ohne dass ein einziger deutscher Chronist uns davon berichtete, nicht einmal der Verfasser des liber de unitate ecclesiae, welcher 1092 oder 1093 seine begeisterte Vertheidigung Heinrichs IV. und des von ihm ernaunteu Gegeupapstes niederschrieb. Dagegen passen die Worte des Cusmas vortrefflich zum Verlauf der Mainzer Synode rom Mai $11) 85 \%$. Hier wurden viele Beschlüsse, ,plurima decreta super statu sanctae ecclesiae", gefasst: Anerkenuung Wiberts als des rechtmüssigen Papste; ${ }^{3}$ ), Fixkommunikation und Absetzung 15 gregorianischer Erzbischüfe und Bischöfe ${ }^{\dagger}$ ). Verkündigung eines allgemeinen Gottesfriedens ${ }^{5}$ ).

Ferner waren auf der Synode (1085) nachweislich alle diejenigen Bischöfe zugegen, welche nach der ron Cosmas mitgetheilten Urkunde vom 29. April die Vereinigung der böhmischen und mährischen Diöcese beschlossen haben sollen: Dietrich ron Verdun, Kourad von Utrecht, Udalrich von Eichstädt, Otto von Regensburg ${ }^{6}$ ) und die drei Erzbischöfe von Mainz, Trier, Köln ${ }^{7}$ ). Hieraus geht aber mit voller

1) M. G. IX 91 .

2) Die Synode sollte auf kaiserlichen Befehl awei Wochen nack dem Osterfest (20. April) eröffnet werden: Liber de unitate eccl. cap. 19 (20) S. 76; ann. ratisbon. fragm. M. G. XIII 49; ann. augustani M. G. III 131.

9) Sigeberti chrou. M. G. VI 365 : ,Heinricus .... exigit ab omnibus, ut Hildibrandi depositionem et Luicberti ordinationem subscripto approbentc.

4) De unitate eccl. If 19,20 ed. W. Schwenkenbecber S. 77. 78; ann. ratisbon. M. G. XIII 49; Bermoldi chron. M. G. V 443; Ekkehardi chron. M. G. VI 205. Die gesta archiep. magdeb. M. G. XIV 404 nennen irrtbümlich das Jahr 1086; inhaltlich stimmen sie mit dem Bericht obiger Quellen zum Jahre 1085 überein.

5) M. G. Leges II $55 \mathrm{ff.;} \mathrm{Ekkehardi} \mathrm{chron.} \mathrm{M.} \mathrm{G.} \mathrm{VI} 206$.

6) Sogar die Reihenfolge der im Diplom M. G. IX 92 genannten Namen gleicht der Aufzählung des liber de unitate eccl. II 19 a. a. O. S. 77, in welchem sich ein ausführliches Verzeiehnis aller $1085 \mathrm{zu}$ Mainz anwesenden Bischöfe findet.

7) Auch Erzbischof Liemar von Bremen, der im Diplom vom 29. April genaunt ist, war nach Cosmas persönlich anwesend. Nach dem liber de unitate eccl. liess er sich dagegen 1085 durch eincn Legaten vertreten. Da Liemar aber im voraus seine Zustimmung zu sämmtlichen Beschlüssen gab, welche dem katholischen Glauben, dem Frieden und der Einheit der Kirche förderlich seien (cf. Liber de un. eccl. II 19 a. a. O. S. 76), konnte der Verfasser des kaiserlichen Diplons ohne Beeinträchtigung der historischen Wabrheit auch Liemar unter den , vier Erzbischöfen anführen, durch welche,primitiva illa parrochia cum omni 
Deutlichkeit hervor, dass in dem Diplom vom 29. April, welches, wie bereits bemerkt, von der Mainzer Synode als von einem historischen Ereignis spricht, nur die Synode des Jahres 1085 gemeint sein kaun. Auch Bischof Gebhard von Prag, der nach Cosmas dem Kaiser zu Mainz 1086 die Klageschrift vorlegte, gehörte zu den Theilnehmern der Synode vom Jahre $1\left(185^{1}\right)$.

Der Inhalt des Diploms vom 29. April 11186, das Itiuerar Heinrichs IV, die inhaltliche Uebereinstimmung der ron Cosmas gegebenen Darstellung mit den Berichten über die Synode von 1085 sprechev dafür, die angebliche Syuode vom Jahre 1086 mit der im Jahre 1055 abgehaltenen zu ideutifizıren. Dazu kommt endlich das bisher überselıene Zeugnis der altenzeller Annalen, wach welcheu die Krönung Wratislavs nicht 1086, wie Cosmas berichtet, sondern auf der Mainzer Synode des Jahres 1085 stattfand: ,Heinricus IlI. Imperator Maguncie in presentia electorum tam spiritualiuı quan secularium principem Wratislaum ducem Bohemie magnifice decoravit-2) etc. Die ziemlich

terminorum suorum ambitu Pragensi sedi est adiudicatac. Aus diesen Worten dee Liploms scheint Cosmal: ('gl. den Text seiner Darstellung in Cap. 37) irrthümlich auf persönliche Anwesenheit Liewars geschlossen zu haben, da er von vier anwesenden Erzbischöfen spricht. Wenn Cosmas ferner nur, 1라 praesulese als Theilnehmer der Synodalverbandlungen bezeichnet, wäbrend der liber de unitate eccl. 16 Bischöte aufzählt, so ist dies von geringem Belang, da abge. sehen von der oftmals sehr flüchtigen Schreibweise des Cosmas bei Lahlen. angaben ein Versehen leichter als sonst geschehen konnte.

1) Liber de unitate ecrl. Il 19 a. a. O. S. 77.

2) M. G. XVI 41. Die ann. bohemici (1)ei Miklosich Slavische Bibliothek II 302) sowohl, als die ann. pragenses M. G. III 120 verlegen die hrönung Wratislav's in das Jahr 1088, die ann. pegavienses M. G. XVI 237 gar in's Jahr 1080 oder 1081. Die in der zweiten Hälfte des 12. Jahrh. rerfassten pegauer Annalen sind besonders in ihrem ersten Theil, der Biographie Wiprechts von Groitsch. durch Irrthümer und sagenhafte Erfindungen fast bis zur Lnbrauch. barkeit. entstellt. Nach den pegauer Anualen ist der Hergang folgender: Heinrich IV,, von den Sacbsen bedrängt, lässt sich durch Wiprecht von Groitsch bereden, Wratislav zu krönen, wenn dieser ihm 4000 Talente übergeben würde und seinen Sohn Bořivoy mit 300 Kriegern am Romzug theilnehmen liesse. Wiprecht meldet dem böhwischen Herzog. was er für ihn erreicht. Dieser erscheint (1080 oder 1081) auf dem Hoftage zu Würzburg (?) in Begleitung angesehener Edlen scines Landes, übergiebt dem Kaiser die versprochene Summe und wird von inm zum Könige gekrönt. - Da Wratislars Krönung nach Ansicht des Annalisten nicht 1085 zu Mainz, sonderu 1080 oder 1081 zu Würzburg geschehen, ist es schwer zu entscheiden, ob Heinrich IV. die angegebene Summe in Wirklichkeit zu Mainz vor der Krönung oder vielmehr 1081 vor dem Romzuge überreicht wurde. Dass die Zahlung der 4000 Talente eine Art Loskaufssumme von der jährlichen Tributpflicht des Böhmenherzogs war, ist eine Vermuthung Palacky's I 320, die W. Tomek Geschichte Böhmens S. 58, Huber Geschichte Oesterreichs 
eingehende Nachricht der ann. veterocellenses, welche nachträglich von einer Haud des 15. Jahrhunderts in den Text der Annalen eingefügt ist, hat neben Cosmas selbständigen Werth.

Dass Cosmas sich in der Chronologie geirrt, kaun selbst dort nicht befremden. wo er als Zeitgenosse berichtet. Von den Reichsangelegenheiteu hat er die dürftigste Kenutnis. Gregor VII. und Heinrich IV. erwähnt er kaum. Die Kämpfe am Regenflusse erzählt er z.um Jahre 1106 (statt 1105) 1). den Römerzag Heiurich's V. (1110-1111) findet man unter der Jahreszahl 11129) u. s. w. Wie der Irrthum des Chronisten entstanden, erklärt sich zudem in einfacher Weise. Seine Quelle in Capitel 37 ist offeubar das von ihm selbst mitgetheilte Diplom gewesun, das er freilich oberfläcblich genug gelesen und stellenweise missverstanden hat ${ }^{3}$ ). Hier fand er deu

$1 \cdot 2 \cdot$ u. a. übernommen haben. Zu ihrer Begründung lässt sich aber nur der Umstand anführen. dass jene Tributpflicht un den Quellenberichten späterer Zeit nirgends mebr erwähnt wirr.

1) Cosmas III 18 M. G. IX 100. Nacb Ekkebards Chronik M. 6. VI 227 finden die Kämpfe am Regenfusse im September oder spätestens in den ersten 'lagen des Oktobers 1105 statt.

?) Cosmas III $38 \mathrm{M}$. G. IX 121.

3) Auftällig ist z. B. der Gebrauch des Wortes, querimonia '. Im Laufe der Lntersuchung ist mehrfach auf wörtliche Anklänge zwischen der Darstellung les Cosmas und dem Text des kaiserlichen Diploms hingewiesen worden; so bietet sich in Capitel 37 besser als an irgend einer anderen Stelle der Chronik die Nöglichkeit, durch Vergleich der im Wortlaut mitgetheilten Quelle und ihrer Verwerthung einen Einblick in die Arbeitsweise des Chronisten und danit «ngleich ein Lirtheil über seine Glaubwürdigkeit auch in Mittheilung zeitgenössischer lireignisse zu gewinnen. Man würde dem verdienten böhmischen Chronisten mit dem Vorwurf absichtlicher Entstellung sielleicht Unrecht thun; zweifellos aber ist die Schilderung tendenziös gefärbt durch den čechisch-nationalen, klerikalen Standpunkt des Verfassers, der in dem unverhohlenen Hass gegen Polen, gegen deutsches Wesen und in der rückhaltslosen Parteinahme für die Interessen des Prager Bisthums zum Ausdruck kommt und gerade im zweiten Buch der Chronik - in dem, was verschwiegen wird nicht minder, als in der Auswahl und Darstellung des gebotenen Stoffes - deutlich erkennbar ist. Drei Ereig. nisse der böhmischen Geschichte stehen hier bezeichnenderweise durchaus im Vordergrund: 1. Bretislavs siegreicher Feldzug gegen Polen mit der Eroberung Ginesen's und der Translation der Adalbertsreliquien nach Prag, 2. die Vertreibung der Deutschen aus Böhmen durch Spithiněv, , ein für alle Jahrhunderte denkwürdiges Ereignis" (Cosmas II 14 M. G. IX 76, II 23 M. G. IX 82; vgl. dazu Loserth, Der Herzog Spithiněv und die angebliche Vertreibung der Deutschen aus Böhmen " in den Mittheil. d. Inst. f. österr. Gesch. Bd. IV 177 ff.), 3. der mährisch.böhmische Kirchenstreit, welcher 1085 zur Vereinigung der Diöcesen Prag und Olmütz führte. Die Wiederherstellung des Prager Bisthums im alten Umfange bildet den Mittelpunkt der Chronik; für den Chronisten hat sie augen- 
29. April 1086 als Datum angeführt. Da er übersah, dass von der Mainzer Synode in dem Diplom als von einem vergangenen Ereiguis gesprochen wird, lag es nahe, die Darstellung der Synodalverhandlungen und die Königskrünung Wratislars dem Jahre 1086 zuzuweisen.

scheinlich das grosste Interesse - es geht dies unter anderem daraus hervor. dass "r hier allein cine urkundliche Quelle im Wortlan mitheilt - : vielleicht sogar hat sie bestimmenten linfuss gehabt auf den Eutschlues des Cosmas. die lieschichte seines Heimatlindes $z$ s scheiben, die urspringlich wohl mit lem neunten Dezemnium des elften Jiarhunderts abschliessen sollte. 